\title{
Spinal Cord Stimulation for Refractary Neuropathic Pain and Its Action on the Neurovegetative Systems
}

\author{
Sara S. Pedrão, Gabriela R. Lauretti, Caio C. M. S. Matias, Raquel de Oliveira, \\ Carlos A. F. Trindade
}

School of Medicine of Ribeirão Preto, University of São Paulo, São Paulo, Brazil

Correspondence to: Gabriela R. Lauretti, grlauret@fmrp.usp.br

Keywords: Spinal Cord Stimulation, Bowel Function, Bladder Function, Neurovegetative Actions

Received: May 31, 2019 Accepted: September 27, $2019 \quad$ Published: September 30, 2019

Copyright $\odot 2019$ by author(s) and Scientific Research Publishing Inc.

This work is licensed under the Creative Commons Attribution International License (CC BY 4.0).

http://creativecommons.org/licenses/by/4.0/

\section{(c) (1) Open Access}

\section{ABSTRACT}

BACKGROUND: Spinal Cord Stimulation (SCS) has been used for chronic refractory pain, however its effectivity on neurovegetative systems has not been evaluated to data. OBJECTIVES: The study was conducted to evaluate the effect of SCS in patients suffering from refractory neuropathic pain, related to analgesia, quality of life and neurovegetative actions on the digestive and urinary tracts. Fifteen patients acted as their own control related to all evaluations. $\mathrm{P}<0.05$ was considered significantly. RESULTS: 13 patients completed the study. The incidence of indication was $6 \%$ in our Center for Pain Treatment in the Teaching Hospital. The SCS improved quality of life, bowel transit and urinary function, as improved overall analgesia and decreased daily rescue analgesic $(\mathrm{p}<0.001)$. CONCLUSIONS: In accordance with literature, the incidence of complications was $20 \%$, and SCS improved analgesia and quality of life. In addition, patients also referred to improved bowel activity and urinary function.

\section{INTRODUCTION}

The physiological mechanisms behind the therapeutic effects of spinal cord stimulation (SCS) are only partially understood. SCS has been shown to increase release of the inhibitory neurotransmitter GABA-A in the spinal cord and decrease glutamate levels. The $\mathrm{GABA}_{\mathrm{B}}$ receptor has been shown to be particularly instrumental in this response [1,2]. Other molecules have also been implicated in the response of SCS to the patient's condition, including activation of the cholinergic system with release of acetylcholine, adenosine, serotonin, and norepinephrine [1]. Although the effect of SCS after spinal cord injury has been recently evaluated regarding colorectal and bladder functions in animals [3] and patients [4-6], its effect on the neurovegetative system in patients submitted to conventional SCS implantation after refractory neuropathic pain or Failed Back Pain Syndrome has not been addressed to date. 
The study was designed to evaluate effectiveness of SCS for chronic refractory neuropathic pain, related to quality of life, analgesia, and neurovegetative (bladder, bowel) improvement.

\section{METHODS}

The Ethics Committee of the Teaching Hospital approved the study protocol (HC-FMRP-USP $\mathrm{N}^{\circ}$. 1430/2010) and patients gave written informed consent. We studied 15 patients with SCS devices which had been implanted to treat neuropathic chronic pain secondary to FBSS. They had been previously referred for continued pain after spinal surgery to the Center for Interventional Pain Treatment of the Teaching Hospital-School of Medicine of RibeirãoPreto, University of São Paulo, for SCS implant. The year of implant of the SCS device ranged from 2010 to 2017. Before indication the SCS implantation, all patients followed the protocol of conservative treatment plus physiotherapy, followed by a sequence of percutaneous blocks such as autonomic block plus neuropathic blocks, as indicated, followed if necessary by radiofrequency ablation and modulation, when indicated. Then when all this former steps failed, the patient was indicated for SCS implantation. In the actual study, two different models of SCS device (MEDTRONIC) had been implanted in our 15 experimental patients. Each patient was prospectively evaluated and acted as his own control.

The patients' characteristics are described in Table 1 . All patients were submitted to conventional stimulation $(60 \mathrm{~Hz} / 350 \mu \mathrm{sec})$ for felling paresthesia in the affected area. At the time of the implant, patients had been complaining of recurring or persistent leg pain and back pain, despite one or more anatomically successful back surgeries for the same original pain and systemic use of daily gabapentin, antidepressants and methadone, as part of our protocol. Exclusion criteria included pregnancy, coagulopathy, severe addiction to psychoactive substances, and lack of ability to cooperate (e.g. due to active psychosis or cognitive impairment).

The demographic data were noted, as much as daily rescue analgesics consumption. Pain and capacity or routine activities were evaluated by VAS pain scores, where zero meant absence of pain or completely able for routine activities; and $10-\mathrm{cm}$ meant worst possible pain or unable for routine activities. The qualities of night sleep, as much as the quality of gastric digestion, bowel and bladder function and urination were evaluated by the criteria of three words: 1) improved, 2) the same, or 3) worst; at the times: 1) before SCS, 1) 1 month, 2) 3-months, 4) 6-months and 5) 12-months after SCS implantation. Any adverse effects were noted and treated as necessary.

\section{STATISTICS}

The power of the study was based upon preliminary data. We hypothesised that the SCS would improve bowel and bladder function by $80 \%-100 \%$ compared to the control. With a beta value of $80 \%$ and an alpha value of 0.05 , these assumptions would require at least 12 patients. $\mathrm{P}<0.05$ was considered significant. Data are expressed as mean $\pm \mathrm{SD}$, unless otherwise stated.

The normality of the data was evaluated by the Shapiro Wilkings test. Demographic data were described. Quality of sleep, daily activities and analgesia time was evaluated by t-student (analgesia) or Wilcoxon Matched Pairs test (sleep and capacity). $\mathrm{P}<0.05$ was considered significant. Adverse effects were

Table 1. Demographic data related to age, weight, height and time of pain complain.

\begin{tabular}{ccccc}
\hline & $\begin{array}{c}\text { Age } \\
\text { (years) }\end{array}$ & $\begin{array}{c}\text { Weight } \\
(\mathrm{Kg})\end{array}$ & $\begin{array}{c}\text { Height } \\
(\mathrm{cm})\end{array}$ & $\begin{array}{c}\text { Time for pain complain } \\
\text { (years) }\end{array}$ \\
\hline Mean & 50.76923 & 69.84615 & 166.0769 & 10.61538 \\
STD & 14.11945 & 14.11764 & 9.473309 & 5.867118 \\
\hline
\end{tabular}

STD-standard deviation of the mean. 
described.

\section{RESULTS}

From the population evaluated, less than $6 \%$ was indicated for SCS in the teaching hospital. Finally, from the 15 patients, 13 were scheduled. One patient was excluded due to infection at the site of the generator, and other was excluded due to the decision at the time of sacral implantation of electrodes. The demographic data is described in Table 1. Table 2 characterizes the religion, gender, race and drugs intake. While Table 3 describes the pathology correlated to each patient.

Related to pain, all patients complained of neuropathic burning, refractory pain. Table 4 describes pain intensity (VAS $\mathrm{cm}$ ) just before and 1-month after the SCS implantation. Neuropathic pain improved more than $70 \%$ after 30 days implantation during all evaluation $(\mathrm{p}<0.01)$. Figure 1 describes VAS pain values during the 12 months assessment. Pain VAS improved after the 1-month, and kept constant during the 3-, 6- and 12-month evaluation $(\mathrm{p}<0.001)$.

Table 2. Demographic data related to religion, gender, race and drugs intake regarding the number of patients.

$\begin{array}{llcl}\text { Religion } & \text { Gender } & \text { Race } & \text { Drugs intake } \\ \text { (C ou E) } & \text { M ou F } & \text { B and W } & \end{array}$

\begin{tabular}{ccccc}
\hline Number of & C-12 & M-6 & W-10 & Antidepressants-13 \\
patients & P-1 & F-7 & B-3 & Amitriptyline-7 \\
& & & & Duloxetine-4, Venlafaxine-2 \\
& & &
\end{tabular}

C-catholic, P-presbiterian; M-male; F-female; W-White, B-Black.

Table 3. Description of gender, related to pathology and area of pain.

\begin{tabular}{cccc}
\hline Patient & Pathology & Gender & Painful area \\
\hline 1 & FBSS & M & Back pain, legs \\
2 & FBSS & F & Back pain, legs \\
3 & CRPS & F & Left arm, cervical \\
4 & CRPS & F & Right arm, cervical \\
5 & Angina + FBSS & M & Thorax, back pain, legs \\
6 & CRPS & F & Right arm, cervical \\
7 & FBSS & F & Back pain, legs \\
8 & FBSS & F & Back pain, legs \\
9 & CRNP & F & Back pain, legs \\
10 & CRNP & M & Thorax, back pain, legs pain \\
11 & CRPS & M & Right arm, cervical \\
12 & FBSS & M & Back pain, legs \\
13 & FBSS & M & Back pain, legs \\
\hline
\end{tabular}

FBSS-fail back surgery syndrome; CRNC-chronic refractory neuropathic pain; CRPS-complex regional pain syndrome. 
Table 4. Pain intensity prior and 1-month after SCS implantation.

\begin{tabular}{ccc}
\hline & Before $($ VAS-cm) & After $($ VAS-cm) \\
\hline mean & 9.076923 & 3.692308 \\
STD & 1.037749 & 0.751068 \\
\hline
\end{tabular}

$\mathrm{P}<0.001$; VAS-pain visual scale $(\mathrm{cm}) ;$ STD-standard deviation.

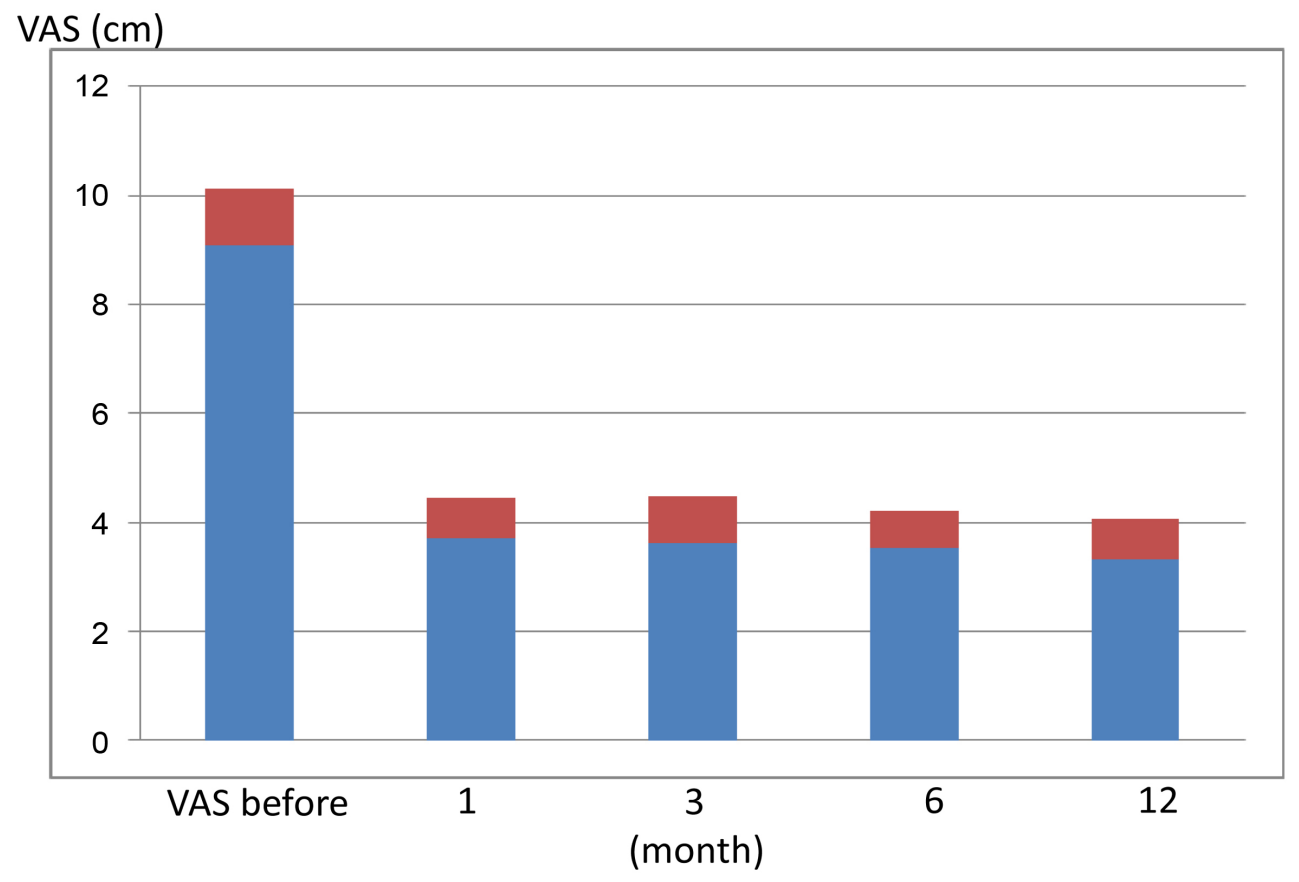

Figure 1. VAS pain values $(0-10 \mathrm{~cm})$ before SCS implantation, and after 1,3 , $6 \mathrm{e} 12$ months implantation. 1 -month $=3$-month $=6$-month $=12$-month $<$ before SCS $(\mathrm{p}<0.001)$.

All patients referred improvement of sleep pattern when before implantation was compared to post-SCS implantation (data not shown, $\mathrm{p}>0.05$ ). Figure 2 describes the evolution of routine activities $(V A S \mathrm{~cm}$ ) just before and 1-month after the SCS implantation. It describes the evolution of improvement of routine activities after SCS implantation. VAS values at 3-, 6- and 12-month after SCS implantation were similar and improved compared to both before and at 1 -month post-SCS implantation $(\mathrm{p}<0.001)$.

Related to gastric digestion, bowel and bladder function, 8 of 13 patients improved after SCS implantation $(\mathrm{p}<0.05)$ (Table 5), and finally, related to adverse effects, one of the patients had electrode migration, in the second patient, there was infection at the site of the generator, and the third patient had to change the percutaneous technique by open surgery, due to obesity.

\section{DISCUSSION}

SCS is a surgical treatment for chronic neuropathic pain refractory to conventional treatment. It consists of one or more leads implanted in the epidural space of the spinal canal, connected to an implantable pulse generator. Each lead carries a number of contacts capable of delivering a weak electrical current to the spinal cord, evoking a feeling of peripheral paresthesia. With correct indication and if implanted by an experienced implanter, success rates generally are in the range of about 50\%-75\%. Among common indications include CRPS, radicular pain after failed back surgery syndrome and refractory neuropathic pain 


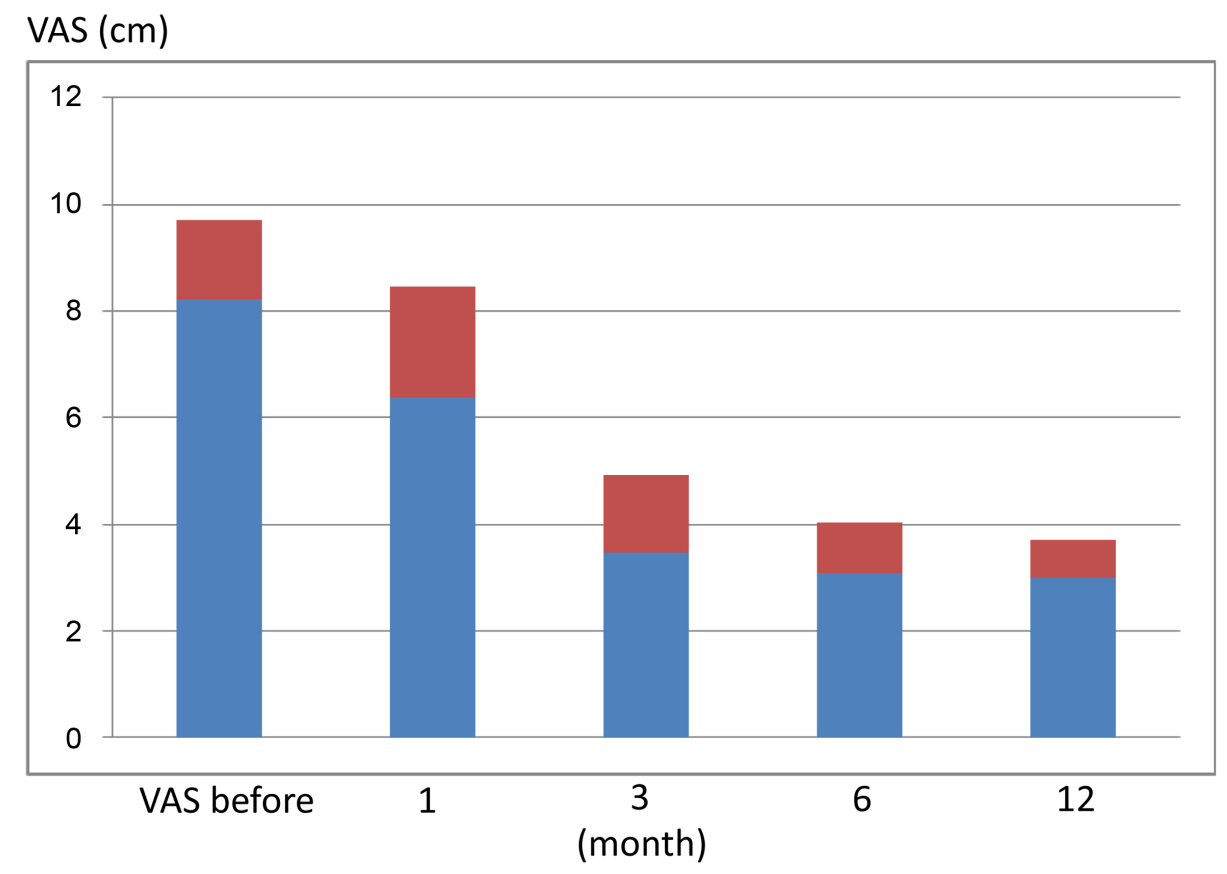

Figure 2. VAS values for routine activities at 3-, 6- and 12-month after SCS implantation. Before $=1-$ month $>3-$ month $=6-$ month $=12-$ month $(\mathrm{p}<0.001)$.

Table 5. Description of gender, related to pathology and neurovegetative function after SCS implantation.

\begin{tabular}{cccc}
\hline Patient & Pathology & Gender & $\begin{array}{c}\text { Gastric digestion, } \\
\text { bladder and bowel } \\
\text { function }\end{array}$ \\
\hline $\mathbf{1}$ & FBSS & $\mathrm{M}$ & Improved \\
$\mathbf{2}$ & FBSS & $\mathrm{F}$ & Improved \\
$\mathbf{3}$ & CRPS & $\mathrm{F}$ & Same \\
$\mathbf{5}$ & CRPS & $\mathrm{F}$ & Same \\
$\mathbf{6}$ & Angina + FBSS & $\mathrm{M}$ & Improved \\
$\mathbf{7}$ & CRPS & $\mathrm{F}$ & Same \\
$\mathbf{8}$ & FBSS & $\mathrm{F}$ & Improved \\
$\mathbf{9}$ & FBSS & $\mathrm{F}$ & Improved \\
10 & CRNP & $\mathrm{F}$ & Improved \\
11 & CRNP & $\mathrm{M}$ & Same \\
12 & CRPS & $\mathrm{M}$ & Same \\
13 & FBSS & $\mathrm{M}$ & Improved \\
\hline
\end{tabular}

$\mathrm{P}<0.05$; FBSS-fail back surgery syndrome; CRNC-chronic refractory neuropathic pain; CRPS-complex regional pain syndrome. 
$[7,8]$, as in our study population. Interesting, the population was constituted by mainly white people. To our knowledge, there is no study about race and indication of SCS.

One point to be addressed is that it was as part of the study protocol, each patient acted as his own control. The possibility of having a control group was discarded because cost, as SCS is not included in the Brazilian health system, and secondly, we believed in a better veracity for scoring subject data, when it was done by the same patient. Related to pain, patients had more than $70 \%$ improvement after 30-day implantation, and kept constant during the 12-month evaluation, in accordance to others $[9,10]$. In our study, conventional stimulation $(60 \mathrm{~Hz} / 350 \mu \mathrm{sec})$ was applied to all patients, consequently, paresthesia was obtained as part of the methodology. Apart from pain relief, all patients referred improvement of sleep pattern when before implantation was compared to post-SCS implantation, in accordance to other studies which suggested potential benefits of SCS on sleep quality in refractory FBSS patients implanted with SCS and enrolled in the French multicenter study [11].

Importantly, the evolution of patients after SCS related to routine activities improved dramatically after the 3-month, and kept constant during all 12-month period evaluation. Although in the present study the improvement of functional capability was demonstrated after the 3-month, Scalone L et al. (2018) described that pain, functional disability and Health Related Quality of Life significantly reached improvement after 6 months from SCS and generally remained stable during the 2-year follow-up [12]. In contrast, our population in this actual study demonstrated an improvement of pain after 1 month, improvement of functional disability after the 3-month. In fact, the good results we showed could be result of less than $6 \%$ indication of SCS implantation, which incidence was not described in other studies.

In the present study, 8 of 13 patients have improved neurovegetative functions. A potential limitation could be the possible low statistical power due to a small study sample. The sample size for the present study was decided according to a clinical effectiveness parameter pain recovery, and probably the number for neurovegetative functions could not be enough, thus not permitting any extrapolation related to the fact that most FBSS had improved neurovegetative data, while only part of CRPS and CNRP had improved. The micturition control, despite the apparent simplicity in its functioning, shows several levels of relative complexity. In conscious and unconscious states, it involves the activity of peripheral nerves, the sacral spinal cord, and the central areas that constitute the medulla oblongata, pons, midbrain, and cerebral cortex. Other central structures such as the cerebral cortex, basal ganglia, and cerebellum have also been evidenced for modulating the micturition [13]. Related to The rationale behind SCS activity at neurovegetative functions is still unknown. Preliminary evidence suggests that transcutaneous tibial nerve stimulation in acute spinal cord injury is able to achieve bladder neuromodulation via modulation of autonomic nervous system functions [14], however it needs further studies. For example, incontinence in women with lumbar spinal canal stenosis is significantly different from the control group. Leakage of urine happened more frequently, the amount of urine leaked was greater, and the general impact on everyday life is harsher, as compared to the clinical control group [15].

In the present study, concomitantly to the bladder function improvement, the bowel mobility was also apparently restored, as much as gastric digestion, in accordance to others who described bowel-bladder synergy improvement in the participants while restoring volitional urination in one with SCS $[6,16]$. The descending serotoninergic raphespinal tract represents new potential therapeutic. The effect of the medullary raphe nuclei on colorectal motility is exerted through activation of 5-hydroxytryptamine type 2 and type 3 receptors in the lumbosacral defecation center [4]. Unfortunately, few studies addressed visceral dysfunction e the SCS utility $[3,6]$.

\section{CONCLUSION}

As conclusions, as no literature reported the effect of SCS on neurovegetative pain, the incidence of complications was $20 \%$, and SCS improved analgesia and quality of life, however, the pain relief started at 1-month and improved capacity for routine activities at 3-month. In addition, patients also referred to improved bowel activity and urinary function, although its mechanism was still unknown. 


\section{FUNDING}

Funding from the Center for Pain Treatment-Teaching Hospital of the School of Medicine of Ribeirão Preto, University of São Paulo.

\section{ACKNOWLEDGEMENTS}

Research funding was partly from the Clinic for Pain Treatment-Teaching Hospital, School of Medicine of Ribeirão Preto, University of São Paulo. The Teaching Hospital-School of Medicine of RibeirãoPreto donated one SCS divide for the study.

\section{CONFLICTS OF INTEREST}

There are no conflicts of interest from any of the authors.

\section{REFERENCES}

1. Chakravarthy, K., Fishman, M.A., Zuidema, X., Hunter, C.W. and Levy, R. (2019) Mechanism of Action in Burst Spinal Cord Stimulation: Review and Recent Advances. Pain Medicine, 20, 13-22. https://doi.org/10.1093/pm/pnz073

2. Jensen, M.P. and Brownstone, R.M. (2019) Mechanisms of Spinal Cord Stimulation for the Treatment of Pain: Still in the Dark after 50 Years. European Journal of Pain, 23, 652-659. https://doi.org/10.1002/ejp.1336

3. Guiho, T., Azevedo-Coste, C., Guiraud, D., Delleci, C., Capon, G., Delgado-Piccoli, N., Bauchet, L. and Vignes, J.R. (2019) Validation of a Methodology for Neuro-Urological and Lumbosacral Stimulation Studies in Domestic Pigs: A Humanlike Animal Model. Journal of Neurosurgery Spine, 30, 1-11. https://doi.org/10.3171/2018.11.SPINE18676

4. Nakamori, H., Naitou, K., Horii, Y., Shimaoka, H., Horii, K., Sakai, H., Yamada, A., Furue, H., Shiina, T. and Shimizu, Y. (2018) Medullary Raphe Nuclei Activate the Lumbosacral Defecation Center through the Descending Serotonergic Pathway to Regulate Colorectal Motility in Rats. The American Journal of Physiology-Gastrointestinal and Liver Physiology, 314, G341-G348. https://doi.org/10.1152/ajpgi.00317.2017

5. Zaer, H., Rasmussen, M.M., Zepke, F., Bodin, C., Domurath, B. and Kutzenberger, J. (2018) Effect of Spinal Anterior Root Stimulation and Sacral Deafferentation on Bladder and Sexual Dysfunction in Spinal Cord Injury. Acta Neurochirurgica, 160, 1377-1384. https://doi.org/10.1007/s00701-018-3557-1

6. Darrow, D., Balser, D., Netoff, T., Krassioukov, A., Phillips, A., Parr, A. and Samadani, U. (2019) Epidural Spinal Cord Stimulation Facilitates Immediate Restoration of Dormant Motor and Autonomic Supraspinal Pathways after Chronic Neurologically Complete Spinal Cord Injury. Journal of Neurotrauma, 36, 2325-2336. https://doi.org/10.1089/neu.2018.6006

7. Dones, I. and Levi, V. (2018) Spinal Cord Stimulation for Neuropathic Pain: Current Trends and Future Applications. Brain Sciences, 8, 138. https://doi.org/10.3390/brainsci8080138

8. Meier, K. (2014) Spinal Cord Stimulation: Background and Clinical Application. Scandinavian Journal of Pain, 5, 175-181. https://doi.org/10.1016/j.sjpain.2014.03.001

9. Kumar, K. and Rizvi, S. (2013) Cost-Effectiveness of Spinal Cord Stimulation Therapy in Management of Chronic Pain. Pain Medicine, 14, 1631-1649. https://doi.org/10.1111/pme.12146

10. Kapural, L., Peterson, E., Provenzano, D.A. and Staats, P. (2017) Clinical Evidence for Spinal Cord Stimulation for Failed Back Surgery Syndrome (FBSS): Systematic Review. Spine (Phila Pa 1976), 42, S61-S66. https://doi.org/10.1097/BRS.0000000000002213

11. Monlezun, O., Lode-Kolz, K., Roulaud, M., Paquereau, J., Ingrand, P., Veyrieras, C., Brandet, C., Guetarni, F., 
Prévost, A., Bataille, B. and Rigoard, P. (2015) A Prospective Study Evaluating Sleep Quality in Failed Back Surgery Syndrome Patients Treated by Multicolumn Spinal Cord Stimulation: Study Design Protocol and Presentation of the Study Population. Neurochirurgie, 61, S117-S124. https://doi.org/10.1016/j.neuchi.2014.06.006

12. Scalone, L., Zucco, F., Lavano, A., Costantini, A., De Rose, M., Poli, P., Fortini, G., Demartini, L., De Simone, E., Menardo, V., Meglio, M., Cozzolino, P., Cortesi, P.A. and Mantovani, L.G. (2018) Benefits in Pain Perception, Ability Function and Health-Related Quality of Life in Patients with Failed Back Surgery Syndrome Undergoing Spinal Cord Stimulation in a Clinical Practice Setting. Health and Quality of Life Outcomes, 16, 68. https://doi.org/10.1186/s12955-018-0887-x

13. Juc, R.U., Colombari, E. and Sato, M.A. (2011) The Importance of the Nervous System in the Control of Voiding and Urine Storage. Arquivos Brasileiros de Ciências da Saúde, 36, 4-9. https://doi.org/10.7322/abcs.v36i1.76

14. Stampas, A., Gustafson, K., Korupolu, R., Smith, C., Zhu, L. and Li, S. (2019) Bladder Neuromodulation in Acute Spinal Cord Injury via Transcutaneous Tibial Nerve Stimulation: Cystometrogram and Autonomic Nervous System Evidence from a Randomized Control Pilot Trial. Frontiers in Neuroscience, 13, 119. https://doi.org/10.3389/fnins.2019.00119

15. Truszczyńska-Baszak, A., Wysocka, E., Starzec, M., Drzał-Grabiec, J. and Tarnowski, A. (2018) Urinary Incontinence Due to Lumbar Spinal Stenosis Causing Disability and Lowering Quality of Life. Journal of Back and Musculoskeletal Rehabilitation, 31, 735-741. https://doi.org/10.3233/BMR-170976

16. Walter, M., Lee, A.H.X., Kavanagh, A., Phillips, A.A. and Krassioukov, A.V. (2018) Epidural Spinal Cord Stimulation Acutely Modulates Lower Urinary Tract and Bowel Function Following Spinal Cord Injury: A Case Report. Frontiers in Physiology, 9, 1816. https://doi.org/10.3389/fphys.2018.01816 